\title{
Jupiter internal structure: the effect of different equations of state ${ }^{\star}$
}

\author{
Y. Miguel $^{1}$, T. Guillot ${ }^{1}$, and L. Fayon ${ }^{2,3}$ \\ ${ }^{1}$ Laboratoire Lagrange, UMR 7293, Université de Nice-Sophia Antipolis, CNRS, Observatoire de la Côte dAzur, \\ Bd de l'Observatoire, CS 34229, 06304 Nice Cedex 4, France \\ e-mail: yamila.miguel@oca.eu \\ 2 Institut de Physique du Globe de Paris, 1 rue Jussieu, 75005 Paris, France \\ 3 AstroParticule et Cosmologie, 10 rue Alice Domon et Leonie Duquet, 75013 Paris, France
}

Received 16 September 2016 / Accepted 11 November 2016

\begin{abstract}
Context. Heavy elements, even though they are a smaller constituent, are crucial to understand the formation history of Jupiter. Interior models are used to determine the amount of heavy elements in the interior of Jupiter, but this range is still subject to degeneracies because of the uncertainties in the equations of state.

Aims. Before Juno mission data arrive, we present optimized calculations for Jupiter that explore the effect of different model parameters on the determination of the core and the mass of heavy elements of Jupiter. We compare recently published equations of state.

Methods. The interior model of Jupiter was calculated from the equations of hydrostatic equilibrium, mass, and energy conservation, and energy transport. The mass of the core and heavy elements was adjusted to match the observed radius and gravitational moments of Jupiter.

Results. We show that the determination of the interior structure of Jupiter is tied to the estimation of its gravitational moments and the accuracy of equations of state of hydrogen, helium, and heavy elements. Locating the region where helium rain occurs and defining its timescale is important to determine the distribution of heavy elements and helium in the interior of Jupiter. We show that the differences found when modeling the interior of Jupiter with recent EOS are more likely due to differences in the internal energy and entropy calculation. The consequent changes in the thermal profile lead to different estimates of the mass of the core and heavy elements, which explains differences in recently published interior models of Jupiter.

Conclusions. Our results help clarify the reasons for the differences found in interior models of Jupiter and will help interpreting upcoming Juno data.
\end{abstract}

Key words. planets and satellites: interiors - planets and satellites: individual: Jupiter - equation of state

\section{Introduction}

The internal structure of Jupiter is estimated with interior models that use observational constraints such as its mass, radius, and gravitational moments, which are derived from measurements made with Pioneer and Voyager (Campbell \& Synnott 1985). The Juno mission is designed to improve our knowledge of the interior of Jupiter and its formation history by a combination of highly accurate measurements of the gravity and magnetic field of Jupiter as well as of the water abundance in the atmosphere.

Models of the internal structure of Jupiter rely on the study of the properties of hydrogen and helium at high pressures (Saumon \& Guillot 2004; Fortney \& Nettelmann 2010; Baraffe et al. 2014). One of the most successful equations of state (EOS) was published by Saumon et al. (1995; SCvH) and has been used in numerous publications on the interior calculations of giant planets. Since 1995, development in numerical techniques allowed a new generation of EOS calculated from $a b$ initio simulations (Nettelmann et al. 2008; Militzer et al. 2008; Militzer 2006, 2009; Caillabet et al. 2011; Nettelmann et al. 2012; Militzer \& Hubbard 2013; Becker et al. 2014). These

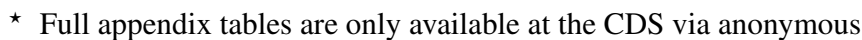
ftp to cdsarc.u-strasbg. fr (130.79.128.5) or via http://cdsarc.u-strasbg.fr/viz-bin/qcat?J/A+A/596/A114
}

equations of state, even though calculated from the same principles and numerical techniques, were used to construct interior models of Jupiter with different results.

While results by Nettelmann et al. (2008) suggested low core masses of up to $8 M_{\text {Earth }}$ consistent with previous estimations (Saumon \& Guillot 2004), results by Militzer et al. (2008) challenged the small core hypothesis, finding large cores of 14-18 $M_{\text {Earth }}$. Nettelmann et al. (2012) improved their previous model and equation of state (Nettelmann et al. 2008), and tested different models for the distribution of heavy elements in the interior of Jupiter. They found that a Jupiter model with a homogenous interior plus a core will lead to larger cores that are more consistent with the estimates of Militzer et al. (2008), while a discontinuous distribution of helium and heavy elements plus a core leads to core masses of up to $8 M_{\text {Earth }}$ but a high mass of heavy elements (28-32 $\left.M_{\text {Earth }}\right)$. Nettelmann and collaborators concluded that the differences in the internal structure of Jupiter originate from different model assumptions, a conclusion in agreement with the analysis of Militzer \& Hubbard (2009). After these papers, two new results were published. Militzer \& Hubbard (2013; MH13) presented a new equation of state for an interacting hydrogen-helium mixture with self-consistent entropy calculations, and a recent paper by Becker et al. (2014; REOS.3) showed updated tables for 
hydrogen and helium in a wide range that covers all temperatures and densities in the interior of Jupiter. These recent estimates still present differences in interior calculations of Jupiter, showing that one of the great challenges in modeling the internal structure of Jupiter still rests on the determination and accuracy of the equations of state of hydrogen and helium.

We explore the differences in the internal structure of Jupiter, on the mass of its derived core and heavy elements, calculated with the same model assumptions but different equations of state. We also explore the effect of different equations of state for heavy elements, different locations of the separation between the molecular and metallic layer, and different models for the heavy element distribution in the interior of Jupiter. In anticipation of Juno measurements, we also study the gravitational moments used to constrain the solutions, to obtain a better knowledge of the sensitivity of the interior of Jupiter to different model parameters and understand the implications of Juno measurements on internal structure calculations.

\section{Modeling Jupiter}

The internal structure of Jupiter is determined from the equations of hydrostatic equilibrium, mass and energy conservation, and energy transport, which are calculated using the code CEPAM (Guillot \& Morel 1995). We set the boundary condition at 1 bar to be $T=165 \mathrm{~K}$ based on Voyager and Galileo measurements (Lindal 1992; Atkinson et al. 1998), where the mass and luminosity are almost equal to the total mass and luminosity of the planet. In this work, we assume that the envelope structure is adiabatic. We note that the presence of deep radiative zones are unlikely (see Guillot et al. 2004). Some recent work included a non-adiabatic, double-diffusive region in the helium demixing region (Nettelmann et al. 2015; Mankovich et al. 2016), but the effect of this on the inferred core mass and mass of heavy elements is significantly smaller than the uncertainties discussed here. We do not consider the possibility that the envelope is entirely double-diffusive, which would yield vastly larger amounts of heavy elements in the interior (Leconte \& Chabrier 2012). We note that dry Ledoux convection tends to homogenize a large part of the envelope (Vazan et al. 2016), implying that this possibility is unlikely.

The helium abundance in the external envelope is taken as $Y=0.238 \pm 0.007$ to match the in situ observations made by the Galileo probe (Zahn et al. 1998). To explain helium depletion compared to the protosolar value $(0.270 \pm 0.005$, Bahcall \& Pinsonneault 1995), we assume that a helium phase transition occurs at a pressure $P_{\text {sep }}$, between 0.8 and 4 Mbar according to the immiscibility calculations of Morales et al. (2013). Helium settles down, increasing the abundance at the deeper layer, which in turn accounts for the depleted amount in the outer envelope. Since the physics and dynamics of helium rain are not understood in detail, we consider two different models for the distribution of solids in the planet interior. In one model helium rain has a fast timescale that allows an efficient mixture of solids in the interior of Jupiter, which has a homogeneous distribution ( $Z$-homogeneous). In the other model we assume that helium rain induces a compositional difference between the two layers, and therefore in this scenario there are two different abundances for the metals in the outer and deeper layer (Z-discontinuous).

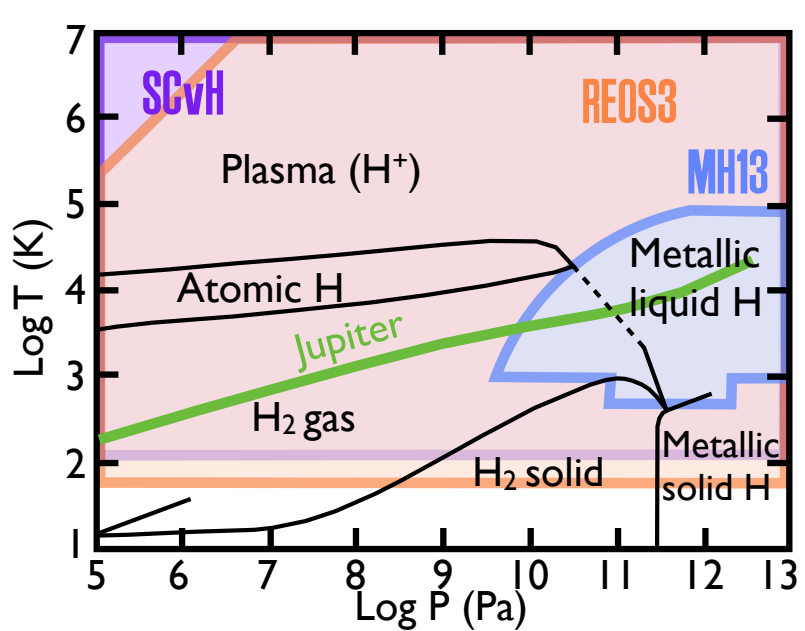

Fig. 1. Phase diagram of hydrogen (adapted from Guillot \& Gautier 2015). The range of validity of each equation of state in the range of the figure is shown in different colors: SCvH is shown in purple, MH13 in blue, and REOS.3 in orange. The internal structure of Jupiter is shown as a green thick line.

\section{Equations of state}

\subsection{Hydrogen and helium}

The proper determination of the internal structure of Jupiter is tied to the accuracy of the equations of state at the range of temperatures and pressures reached in the interior of this giant. Since $\sim 85 \%$ of the Jupiter mass is hydrogen and helium, the equations of state of these elements determine its internal structure. Nevertheless, we show that the treatment adopted for the heavy elements also affects the core mass and total mass of heavy elements that are retrieved with our calculations (Sect. 4.2.2).

In this study we use three different equations of state for hydrogen and helium: the widely used Saumon et al. (1995) equations of state, and the more recent equations of state derived from ab initio calculations published by Militzer \& Hubbard (2013) and by Becker et al. (2014). Figure 1 shows the phase diagram of hydrogen and the range of pressure and temperature covered by each equation of state.

\subsubsection{Pure hydrogen equation of state from results of $\mathrm{MH} 13$}

The table presented by MH13 lists pressure, internal energy, Helmholtz free energy, and specific entropy as functions of density and temperature, while CEPAM uses tables where entropy and density are given as functions of pressure and temperature. We use cubic spline interpolation to create a table in CEPAM format.

As shown in Fig. 1, the MH13 EOS was made for a small range of pressure and temperature that does not cover all pressures and temperatures in the interior of Jupiter. We extend the table using the $\mathrm{SCvH}$ EOS for those temperatures and pressures with no data. To smoothen the limits between the two tables, we use linear interpolation. The new table covers a range of pressures between $10^{4}$ and $10^{19} \mathrm{~g} /\left(\mathrm{cm} \mathrm{s}^{2}\right)$ and a range of temperatures $2.25 \leq \log (\mathrm{T}) \leq 7 \mathrm{~K}$.

The MH13 EOS was made for a mixture of hydrogen and helium $\left(Y_{\mathrm{MH} 13}=0.245\right)$. To allow a change in the composition of the molecular and metallic envelopes, we extracted the hydrogen from the table, creating a pure hydrogen equation of state based on MH13 results. We calculated density and entropy for each pressure and temperature in the table using the equations for a 
mixture and the $\mathrm{SCvH}$ equation of state for helium:

$\frac{1}{\rho_{\mathrm{H}}}=\frac{1}{X_{\mathrm{MH} 13}}\left(\frac{1}{\rho_{\mathrm{MH} 13}}-\frac{Y_{\mathrm{MH} 13}}{\rho_{\mathrm{SCVH}, \mathrm{He}}}\right)$

$S_{\mathrm{H}}=\frac{1}{X}\left(S_{\mathrm{MH} 13}-Y S_{\mathrm{SCvH}, \mathrm{He}}\right)$,

with $\rho_{\mathrm{H}}$ and $S_{\mathrm{H}}$ the density and entropy of the pure hydrogen equation of state we extracted from the table of MH13, $\rho_{\mathrm{SCvH}, \mathrm{He}}$ and $\mathrm{S}_{\mathrm{SCvH}, \mathrm{He}}$ the density and entropy in the helium table of $\mathrm{SCvH}, \mathrm{X}_{\mathrm{MH} 13}, \rho_{\mathrm{MH} 13}$ and $\mathrm{S}_{\mathrm{MH} 13}$ the hydrogen mass fraction, density, and entropy of MH13. Equation (2) neglects the entropy of mixing. Detailed calculations using the SCvH EOS with and without this entropy of mixing show that this is a much smaller effect than the uncertainties on the EOSs themselves discussed here. We call this new hydrogen table MH13+SCvH (shown in Appendix A).

\subsubsection{Entropy calculation for hydrogen and helium using REOS.3}

REOS.3 is a density-temperature equation of state with pressure and specific internal energy that covers a wide range in pressure and temperature (Fig. 1, for hydrogen). To allow comparisons between the tables and avoid errors in the entropy calculation, we changed the zero point of the specific internal energy in the REOS. 3 tables to make them coincide in the ideal gas regime with the SCvH EOS (N. Nettelmann and A. Becker, priv. comm.). Since the difference between the specific internal energy of REOS.3 and SCvH equations of state at $T=60 \mathrm{~K}$ and $\rho=10^{-3} \mathrm{~g} / \mathrm{cm}^{3}$ is $\Delta u_{\mathrm{H}}=1590.12135$ for hydrogen and $\Delta u_{\mathrm{He}}=1843.06795$ for helium, we added these values to all the specific internal energies in the REOS.3 H and He tables, respectively. Figure 2 shows a comparison between the internal energies of SCvH and REOS. $3+\Delta_{u}$.

The entropy is a necessary parameter in internal structure calculations. The two layers considered in the model follow an adiabat, therefore the ratio between the derivatives of the entropy with respect to pressure and temperature gives us the temperature gradient in the planet interior. We calculate the specific entropy, s, for each point of the REOS.3 table through thermodynamic relations between the published $u, P, T$, and $\rho$ (Nettelmann et al. 2012). From the definition of the Helmholtz free energy,

$F=U-T S$,

it follows

$s(T, V)=\frac{u(T, V)}{T}-\frac{1}{M}\left(\frac{F(T, V)}{T}-\frac{F\left(T_{0}, V_{0}\right)}{T_{0}}\right)+s_{0}$.

Since

$\frac{1}{M}\left(\frac{F(T, V)}{T}-\frac{F\left(T_{0}, V_{0}\right)}{T_{0}}\right)=\frac{1}{M} \int_{T_{0}, V_{0}}^{T, V} \mathrm{~d}\left(\frac{F\left(T^{\prime}, V^{\prime}\right)}{T^{\prime}}\right)$

and

$\mathrm{d}\left(\frac{F\left(T^{\prime}, V^{\prime}\right)}{T^{\prime}}\right)=\frac{\mathrm{d} F}{T}-\frac{F}{T^{2}} \mathrm{~d} T$

it follows from Eq. (3) that

$\frac{\mathrm{d} F}{T}=\frac{\mathrm{d}(U-T S)}{T}=\frac{\mathrm{d} U}{T}-\mathrm{d} S-\frac{S}{T} \mathrm{~d} T$ and

$\frac{F}{T^{2}} \mathrm{~d} T=\frac{(U-T S)}{T^{2}} \mathrm{~d} T$.

Then Eq. (6) can be written as

$\mathrm{d}\left(\frac{F\left(T^{\prime}, V^{\prime}\right)}{T^{\prime}}\right)=\frac{\mathrm{d} U}{T}-\mathrm{d} S-\frac{U}{T^{2}} \mathrm{~d} T$,

using that

$\frac{\mathrm{d} U}{T}=-\frac{P}{T} \mathrm{~d} V+\mathrm{d} S$,

then

$\mathrm{d}\left(\frac{F\left(T^{\prime}, V^{\prime}\right)}{T^{\prime}}\right)=-\frac{P}{T} \mathrm{~d} V-\frac{U}{T^{2}} \mathrm{~d} T$.

Now, in the $\rho$ and $T$ plane,

$\frac{1}{M} \mathrm{~d}\left(\frac{F\left(T^{\prime}, \rho^{\prime}\right)}{T^{\prime}}\right)=\frac{P}{T} \frac{1}{\rho^{2}} \mathrm{~d} \rho-\frac{u}{T^{2}} \mathrm{~d} T$.

Finally,

$\frac{1}{M} \int_{T_{0}, \rho_{0}}^{T, \rho} \mathrm{d}\left(\frac{F\left(T^{\prime}, \rho^{\prime}\right)}{T^{\prime}}\right)=\int_{\rho_{0}}^{\rho} \frac{P\left(T_{0}, \rho^{\prime}\right)}{T_{0}} \frac{1}{\rho^{\prime 2}} \mathrm{~d} \rho^{\prime}-\int_{T_{0}}^{T} \frac{u\left(T^{\prime}, \rho\right)}{T^{\prime 2}} \mathrm{~d} T^{\prime}$,

and returning to Eq. (4),

$s(T, \rho)=\frac{u(T, V)}{T}-\left[\int_{\rho_{0}}^{\rho} \frac{P\left(T_{0}, \rho^{\prime}\right)}{T_{0}} \frac{1}{\rho^{\prime 2}} \mathrm{~d} \rho^{\prime}-\int_{T_{0}}^{T} \frac{u\left(T^{\prime}, \rho\right)}{T^{\prime 2}} \mathrm{~d} T^{\prime}\right]+s_{0}$.

The specific entropy at each point is calculated from Eq. (14), using the trapezoid rule for the numerical integration and cubic splines interpolation to add temperature and density points to improve the numerical calculation. Figure 3 shows a comparison of the entropy calculated at different temperatures with other equations of state.

These new equations of state with entropy and internal energies that coincide with $\mathrm{SCvH}$ at $T=60 \mathrm{~K}$ and $\rho=10^{-3} \mathrm{~g} / \mathrm{cm}^{3}$ are called REOS3b (see Appendix A).

\subsection{Comparison with experiments}

The original equations of state of MH13 and REOS.3 experienced some changes, such as the creation of a pure hydrogen table and the extension of this table for a wide pressure and temperature range $\left(\mathrm{MH} 13+\mathrm{SCvH}\right.$, Sect. 3.1.1), the change of the $\mathrm{u}_{0}$ , and the entropy calculation (REOS3b Sect. 3.1.2), and interpolation to add more points and make a pressure-temperature table (MH13+SCvH and REOS3b). To test our final tables, we make comparisons with high-pressure experiments.

Much attention has been devoted to experiments designed to understand the properties of hydrogen (or deuterium) and helium at high densities (Nellis et al. 1983, 1984; Holmes et al. 1995; Collins et al. 1998; Belov et al. 2002; Boriskov et al. 2003; Grishechkin et al. 2004; Knudson et al. 2004; Eggert et al. 2008; Hicks et al. 2009; Celliers et al. 2010; Loubeyre et al. 2012). In these experiments a gas at rest with an initial thermodynamic state $\left(u_{0}, \rho_{0}, P_{0}\right)$ is exposed to an abrupt change in pressure, temperature, and density. Applying the laws of conservation of mass, 

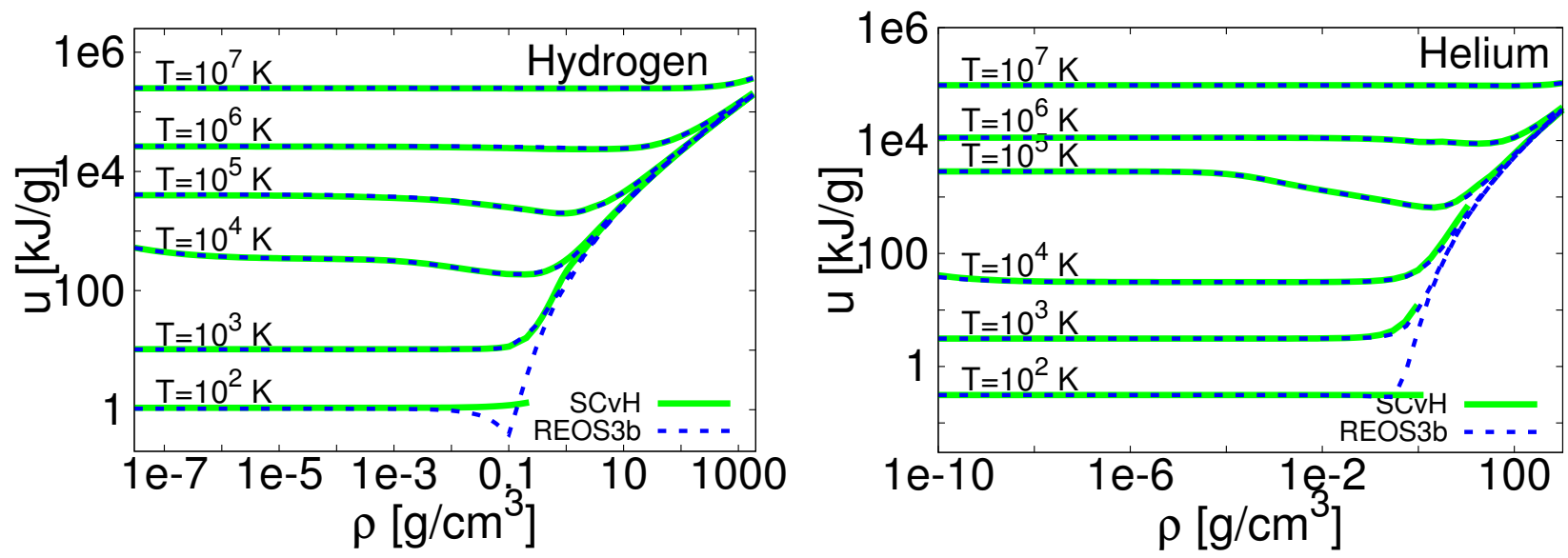

Fig. 2. Specific internal energy as a function of density at different temperatures for hydrogen (left panel) and helium (right panel), using two different equations of state. $\mathrm{SCvH}$ is shown as green solid lines, and the values shown as blue dotted lines correspond to the u in REOS.3 plus $\Delta u$ $\left(\Delta u_{\mathrm{H}}=1590.12135\right.$ for hydrogen and $\Delta u_{\mathrm{He}}=1843.06795$ for helium $)$ or REOS3b.
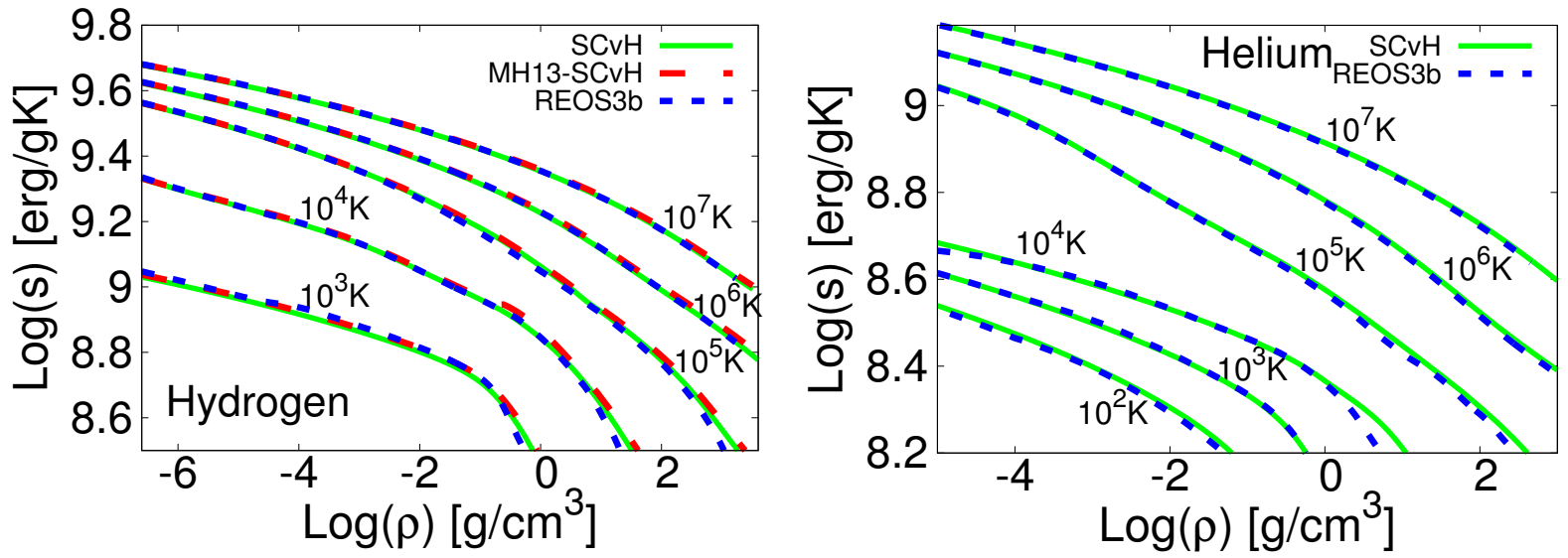

Fig. 3. Specific entropy vs. density at different temperatures for hydrogen (left panel) and helium (right panel). For hydrogen we show a comparison between the entropy calculated with REOS3b (blue), the one published in $\mathrm{SCvH}$ (green), and $\mathrm{MH} 13+\mathrm{SCvH}$ values (red). Since $\mathrm{MH} 13+\mathrm{SCvH}$ is a pure hydrogen table, the right panel shows a comparison between REOS3b and SCvH only.

momentum, and energy at both sides of this shock wave, we derive a relation between the state of the gas before and after the shock, which is called the Rankine-Hugoniot equation:

$H(\rho, P)=u-u_{0}+\frac{1}{2}\left(P+P_{0}\right)\left(\frac{1}{\rho}-\frac{1}{\rho_{0}}\right)$,

where $\rho, P$, and $u$ are the density, pressure, and internal energy of the final shocked gas. Equation (15) defines all states on the $(u$, $\rho, P)$ surface that can be reached from the initial condition by a single shock.

\subsubsection{Hugoniot-curve calculation from $\mathrm{P}, \mathrm{T}, \rho$, and $\mathrm{s}$}

The Hugoniot curve, $H(\rho, P)$, is defined by

$H(\rho, P)=0$.

Since our EOS tables give us $P, T, \rho$, and s, we wish to write Eq. (16) as a function of these variables. If we differentiate Eq. (16), we obtain

$\mathrm{d} H=\mathrm{d} u+\frac{1}{2}\left[\left(\frac{1}{\rho}-\frac{1}{\rho_{0}}\right) \mathrm{d} P-\left(\frac{1}{\rho^{2}}\left(P+P_{0}\right) \mathrm{d} \rho\right)\right]=0$.

Now we know that

$\mathrm{d} u=-P \mathrm{~d} V+T \mathrm{~d} s$ where $V=\frac{1}{\rho}$, and therefore

$\mathrm{d} V=-\frac{\mathrm{d} \rho}{\rho^{2}}$.

Using Eqs. (18) and (19) in Eq. (17),

$\mathrm{d} H=\frac{1}{2}\left(\frac{1}{\rho}-\frac{1}{\rho_{0}}\right) \mathrm{d} P+\frac{1}{2} \frac{\left(P-P_{0}\right)}{\rho^{2}} \mathrm{~d} \rho+T \mathrm{~d} s=0$.

To integrate in the $P, T$ plane, we use

$\mathrm{d} \rho(P, T)=\frac{\partial \rho(P, T)}{\partial P} \mathrm{~d} P+\frac{\partial \rho(P, T)}{\partial T} \mathrm{~d} T$
$\mathrm{~d} s(P, T)=\frac{\partial s(P, T)}{\partial P} \mathrm{~d} P+\frac{\partial s(P, T)}{\partial T} \mathrm{~d} T$.

Equation (20) is written as

$\mathrm{d} H(P, T)=\frac{1}{2}\left(\frac{1}{\rho(P, T)}-\frac{1}{\rho_{0}}\right) \mathrm{d} P+\frac{1}{2} \frac{\left(P-P_{0}\right)}{\rho(P, T)^{2}} \frac{\partial \rho(P, T)}{\partial P} \mathrm{~d} P$
$+\frac{1}{2} \frac{\left(P-P_{0}\right)}{\rho(P, T)^{2}} \frac{\partial \rho(P, T)}{\partial T} \mathrm{~d} T+T \frac{\partial s(P, T)}{\partial P} \mathrm{~d} P+T \frac{\partial s(P, T)}{\partial T} \mathrm{~d} T$ 


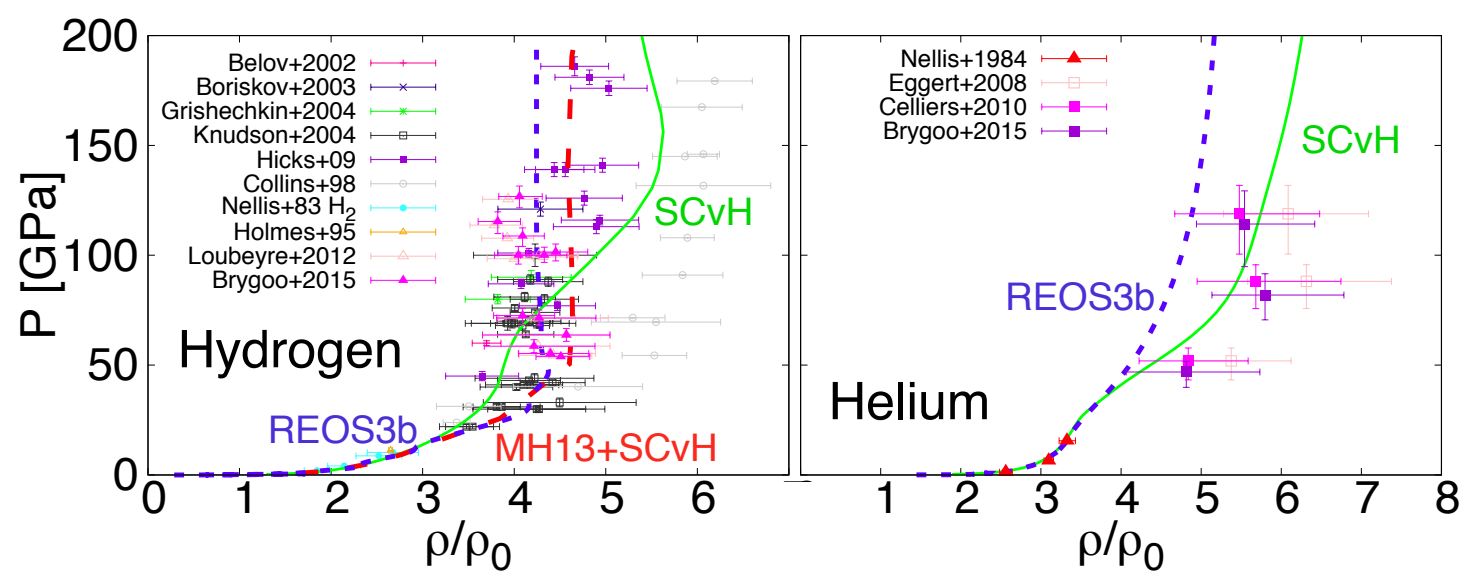

Fig. 4. Principal Hugoniot of hydrogen (left panel) and helium (right panel). The curves were calculated for an initial state of $\rho_{0}=0.0855 \mathrm{~g} / \mathrm{cm}^{3}$ and $T_{0}=20 \mathrm{~K}$ for hydrogen and $\rho_{0}=0.123 \mathrm{~g} / \mathrm{cm}^{3}$ and $T_{0}=4 \mathrm{~K}$ for helium. Experimental results are shown with different point styles for comparison. We included recent estimates by Brygoo et al. (2015), who presented corrections of previously published data on He (Eggert et al. 2008; Celliers et al. 2010), $\mathrm{H}_{2}$, and $\mathrm{D}_{2}$ (Loubeyre et al. 2012) based on a better understanding of shocked compressed $\mathrm{SiO}_{2}$.

Integrating Eq. (23) between an initial point and the final state, we obtain the Hugoniot curve as a function of the variables present in our EOS tables:

$H(P, T)-H_{0}=\frac{1}{2} \int_{P\left(H_{0}\right)}^{P}\left(\frac{1}{\rho\left(P, T\left(H_{0}\right)\right)}-\frac{1}{\rho_{0}}\right) \mathrm{d} P$

$+\frac{1}{2} \int_{P\left(H_{0}\right)}^{P} \frac{\left(P-P_{0}\right)}{\rho\left(P, T\left(H_{0}\right)\right)^{2}} \frac{\partial \rho\left(P, T\left(H_{0}\right)\right)}{\partial P} \mathrm{~d} P$

$+\int_{P\left(H_{0}\right)}^{P} T\left(H_{0}\right) \frac{\partial s\left(P, T\left(H_{0}\right)\right)}{\partial P} \mathrm{~d} P$

$+\frac{1}{2} \int_{T\left(H_{0}\right)}^{T} \frac{\left(P-P_{0}\right)}{\rho(P, T)^{2}} \frac{\partial \rho(P, T)}{\partial T} \mathrm{~d} T+\int_{T\left(H_{0}\right)}^{T} T \frac{\partial s(P, T)}{\partial T} \mathrm{~d} T$.

To find the zeros in Eq. (24), we calculate $H(P, T)$ at each $P$ and $T$ in the EOS table, and when it changes sign, we perform a cubic spline interpolation in $P$ and $T$ to find the exact values of $P$, $T, \rho(P, T)$ and $\mathrm{s}(P, T)$ that will give us $H(P, T)=0$. Figure 4 shows Hugoniot curves for hydrogen and helium obtained when using different equations of state and compared with experimental data.

\subsection{Heavy elements}

Hydrogen and helium are the most relevant species, but an accurate description of the interior of Jupiter needs a definition of the heavy elements equation of state. In our model heavy elements are water and rocks, and we use three different equations of state to test their sensitivity. Following Saumon \& Guillot (2004), we use for rocks the equation of state for a mixture of silicates called "dry sand" in SESAME (Lyon \& Johnson 1992). For water we use the SESAME EOS (Lyon \& Johnson 1992) and a more recent equation of state calculated in Valencia et al. (2013), which combines an equation of state for water at high temperatures $(T>1000 \mathrm{~K})$ (French et al. 2009) with results taken from the NIST database (Saul \& Wagner 1989).

\section{Results}

\subsection{Different thermal structures}

In this section we compare the interior of Jupiter with different equations of state. Figure 5 shows that REOS3b leads to higher

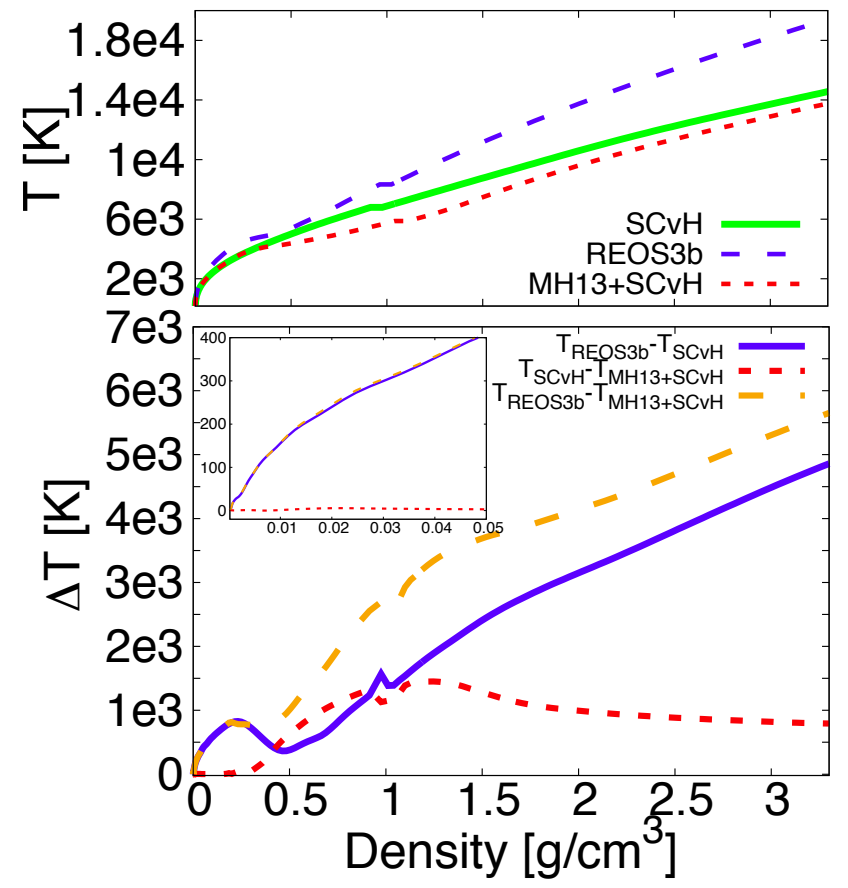

Fig. 5. Top panel: Jupiter density and temperature for different equations of state: $\mathrm{SCvH}$ (green solid), REOS3b (blue dashed), and $\mathrm{MH} 13+\mathrm{SCvH}$ (red dotted line). The discontinuity is due to the separation of the outer and deeper envelope at $P_{\text {sep }}=1$ Mbar. Bottom panel: differences in the temperature obtained with the three different equations of state. The blue line is the temperature difference between REOS3b and $\mathrm{SCvH}$, the red dotted line is the difference between $\mathrm{SCvH}$ and $\mathrm{MH} 13+\mathrm{SCvH}$, and the orange dashed line is the difference between REOS $3 b$ and $\mathrm{MH} 13+\mathrm{SCvH}$.

temperatures for all densities than the other two equations of state. The differences are large even at relatively low densities, being close to $1000 \mathrm{~K}$ for $\rho \simeq 0.2 \mathrm{~g} / \mathrm{cm}^{3}$. Since MH13+SCvH uses $\mathrm{SCvH}$ equation of state for densities $\rho<0.22246 \mathrm{~g} / \mathrm{cm}^{3}$, the differences between these two EOS arise for high densities, where $\mathrm{MH} 13+\mathrm{SCvH}$ reaches lower temperatures. These differences in the thermal profiles explain the different mass of metals in the envelope and mass of the core derived with the optimized models. 
Table 1. Gravitational moments we explored.

\begin{tabular}{ccccc}
\hline \hline$J_{2}\left[\times 10^{-2}\right]$ & $J_{4}\left[\times 10^{-4}\right]$ & $J_{6}\left[\times 10^{-5}\right]$ & Note & Reference \\
\hline $1.4697(0.0001)$ & $-5.84(0.05)$ & $3.10(2)$ & Pre-Juno observed $J_{S}$ & Campbell \& Synnott (1985) \\
$1.4682(0.0001)$ & $-5.80(0.05)$ & $3.04(2)$ & $J_{S}$ with differential rotation & Guillot $(1999)$ \\
$1.469643(0.000021)$ & $-5.8714(0.0168)$ & $3.425(0.522)$ & JUP230 orbit solution & Jacobson $(2003)$ \\
$1.469562(0.000029)$ & $-5.9131(0.0206)$ & $2.078(0.487)$ & JUP310 orbit solution & Jacobson $(2013)$ \\
\hline
\end{tabular}

\subsection{Optimized models}

We calculated optimized models of Jupiter, in which the abundance of heavy elements and the mass of the core $\left(M_{\text {core }}\right)$ are adjusted to reproduce the observables within their error bars (see Guillot et al. 1994, for more details on the method).

\subsubsection{Gravitational moments of Jupiter}

Our models match the radius and gravitational moments $J_{2}$ and $J_{4}$ of Jupiter. These last ones have changed with time according to our improved knowledge of the Jupiter gravity field. Table 1 shows the gravitational moments adopted in this paper. We consider gravitational moments derived from pre-Juno observations by Voyager 1 and 2, Pioneer 10 and 11 (Campbell \& Synnott 1985), as well as more recent values derived from JUP230 and JUP310 orbit solutions ${ }^{1}$, and also values with a correction by differential rotation effects, where the solution of Hubbard (1982) to the planetary figure problem was adopted for a deep rotation field with cylindrical symmetry (Guillot 1999).

Our calculation of the gravitational moments is based on the theory of figures of fourth order. A comparison with more detailed calculations made with a concentric Maclaurin spheroid (Hubbard 2012, 2013; W. B. Hubbard, N. Movshovitz, priv. comm.) showed that our approximation leads to an error on the order of $1 \mathrm{e}-7$ in $J_{4}$ and 2e-6 in $J_{6}$. Figure 6 shows gravitational moments of order 4 and 6 as well as the resulting $J_{\mathrm{S}}$ in all our optimized models with different equations of state. The black arrow shows the error in the determination of $J_{6}$. The observed $J$ s change when considering differential rotation (indicated with the gray arrow in the figure). Further studies including interior dynamics will help improve our understanding of the Jupiter interior from gravity measurements (Kaspi et al. 2010; Galanti \& Kaspi 2016).

The results of our simulations are very confined in the $J_{4}-J_{6}$ diagram, especially in the case of $J_{6}$, which is narrowly defined within this framework. We find larger $\left|J_{4}\right|$ and $J_{6}$ than observed values and the most recent estimates of 2013. Our results with $\mathrm{MH} 13+\mathrm{SCvH}$ and a recent estimate by Hubbard \& Militzer (2016) show a similar tendency toward preferred $J_{4}$ and $J_{6}$ values.

In our models $Y_{\text {deep }}$ is calculated to account for the missing helium in the atmosphere of Jupiter with respect to the protosolar value (Sect. 2). Figure 7 shows $J_{4}, J_{6}$, and $M_{Z}$ found in our optimized models when changing $Y_{\text {proto }}$ and maintaining $Y_{\text {atm }}$ fixed, to test the effect of changing the abundance of helium in the deep layer of Jupiter. To satisfy the constraint in $J_{2}$, larger $Y_{\text {deep }}$ leads to a lower mass of heavy elements in the envelope, which decreases approximately $5 M_{\text {Earth }}$ when going from $Y_{\text {deep }}=0.238$ to $Y_{\text {deep }}=0.28$ in all cases. A larger abundance of helium in the deep layer ensures solutions closer to current $J_{4}$ and $J_{6}$ estimates.

\footnotetext{
1 Values calculated by R. A. Jacobson in 2003 and 2013, respectively, and published at the JPL website: http://ssd.jpl.nasa. gov/?gravity_fields_op
}

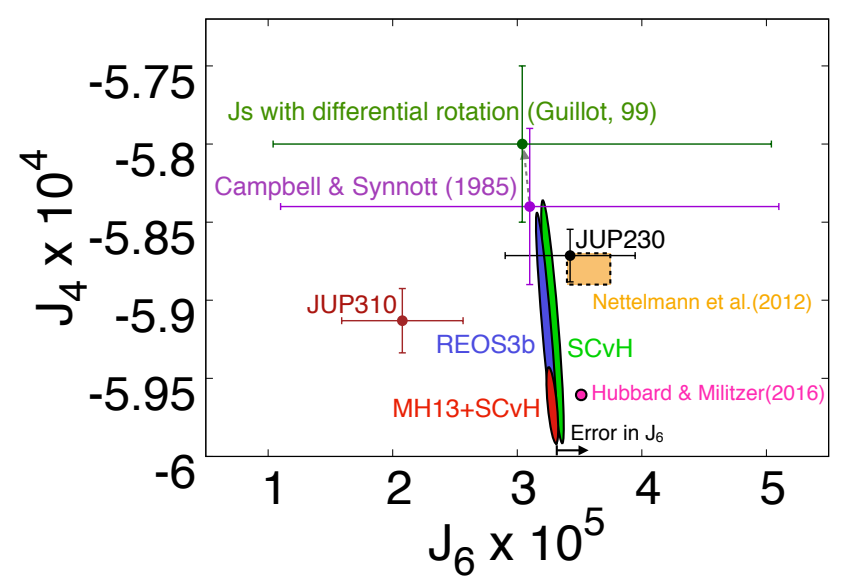

Fig. 6. $J_{4}$ and $J_{6}$ pre-Juno observed values (Campbell \& Synnott 1985 purple), those with a correction due to differential rotation (Guillot 1999; dark green), and more recent estimates by Jacobson in 2003 (black) and 2013 (brown). Js solutions of our optimized models within $2 \sigma$ of Campbell \& Synnott (1985) and modeled with Z-discontinuous are shown in different colors according to the equation of state used in the simulation: $\mathrm{SCvH}$ (green), REOS3b (blue), and $\mathrm{MH} 13+\mathrm{SCvH}$ (red). The pink dot shows a recent model by Hubbard \& Militzer (2016) and the orange box shows estimates by Nettelmann et al. (2012) for comparison.

The mass of the core and the mass of heavy elements found in our models depend on the $J \mathrm{~S}$ used to constrain the solutions. Figure 8 shows that solutions found with $J_{\mathrm{s}}$ derived from observations published by Campbell \& Synnott (1985) lead to larger $M_{\text {core }}$ and smaller $M_{Z}$ than the values found with more recent estimates by Jacobson $(2003,2013)$. $M_{\text {core }}$ estimates derived using $J_{\mathrm{s}}$ by Campbell \& Synnott (1985) reach core masses $4 M_{\text {Earth }}$ higher than the values found with $J_{\mathrm{S}}$ by Jacobson (2003) for REOS3b and SCvH. The lowest $M_{Z}$ find with $J$ s by Campbell \& Synnott (1985) are $6 M_{\text {Earth }}$ lower than estimates found with values calculated by Jacobson (2003) for REOS3b and $\mathrm{SCvH}$. Results found with $\mathrm{MH} 13+\mathrm{SCvH}$ do not change significantly for $M_{Z}$, but there is a difference of $2 M_{\text {Earth }}$ in $M_{\text {core }}$ in the solutions estimated with the different $J_{\mathrm{s}}$. There are no solutions found within $2 \sigma$ with $J$ s estimated by Jacobson (2003). New information provided by Juno will contribute to more accurate data to calculate gravitational moments of higher order and improve the uncertainty in lower orders, toward a better determination of the internal structure of Jupiter.

\subsubsection{Jupiter core and mass of heavy element}

For the following optimized models we adjusted our solutions to reproduce the radius, $J_{2}$ and $J_{4}$ of Jupiter. For $Z$-homogeneous cases we adjusted the core mass and heavy element mass mixingratio, while for $Z$-discontinuous we found the difference between the abundance of heavy elements in the outer and deeper envelope $(\Delta Z)$ and the core mass that best reproduce the observables. 


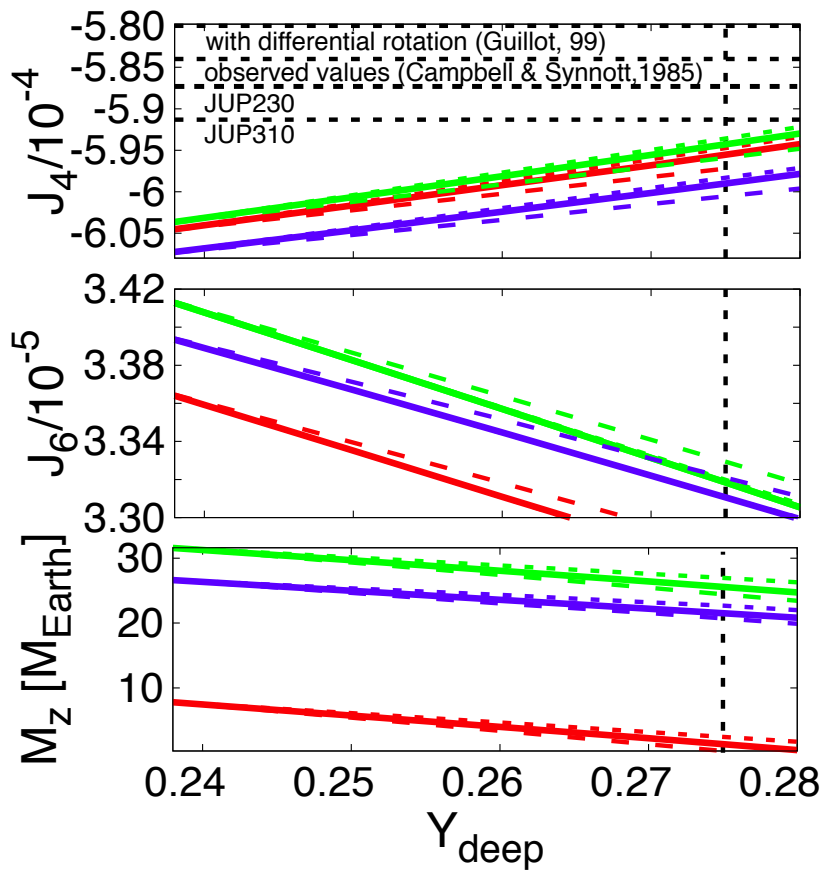

Fig. 7. Model results when adjusting the mass of the core and heavy elements to reproduce the radius and $J_{2}$ of Jupiter. In these models the atmospheric helium mass mixing-ratio is fixed to $Y_{\text {atm }}=0.238$, and we change the helium abundance in the deeper layer to match the protosolar value within its error bars. Different panels show $J_{4}$ (upper panel), $J_{6}$ (middle panel), and $M_{Z}$ (lower panel). Different colors show results for the three different equations of state of hydrogen and helium: $\mathrm{SCvH}$ (green), MH13+SCvH (red), and REOS3b (blue). The lines indicate different locations of the helium phase that separates the two envelopes at $0.8 \mathrm{Mbar}$ (dashed), $2 \mathrm{Mbar}$ (solid), and 4 Mbar (dotted lines). The vertical dashed line indicates the protosolar helium mixing ratio, and the horizontal lines in the upper panel show estimates of $J_{4}$ from observations and models as a reference.

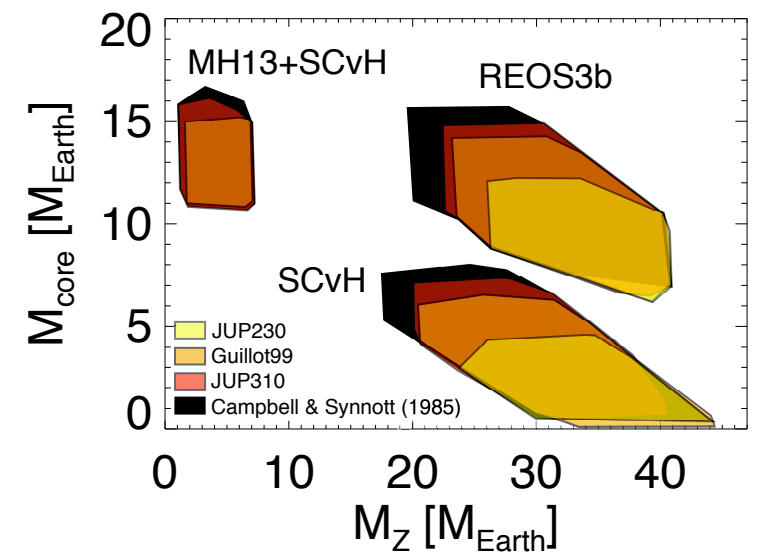

Fig. 8. Mass of the core and heavy elements in the interior of Jupiter derived with different $J$ s. Models use $Z$-discontinuous scenario, $P_{\text {sep }}=$ 2 Mbar and different EOS indicated in the figure. Colored areas show solutions within $2 \sigma$ from the radius, $J_{2}$ and $J_{4}$ of Jupiter estimated by Campbell \& Synnott (1985; black), Guillot (1999; orange), Jacobson (2003; yellow), and Jacobson (2013; red). No solution was found with $\mathrm{MH} 13+\mathrm{SCvH}$ and constraints by JUP230.

Our baseline models were made using $J_{2}$ and $J_{4}$ derived from observations of Jupiter gravity field (Campbell \& Synnott 1985), $P_{\text {sep }}=2$ Mbar and the NIST equation of state for hot $\mathrm{H}_{2} \mathrm{O}$ as the equation of state for heavy elements. Models that differ from these conditions are indicated in the text and figure

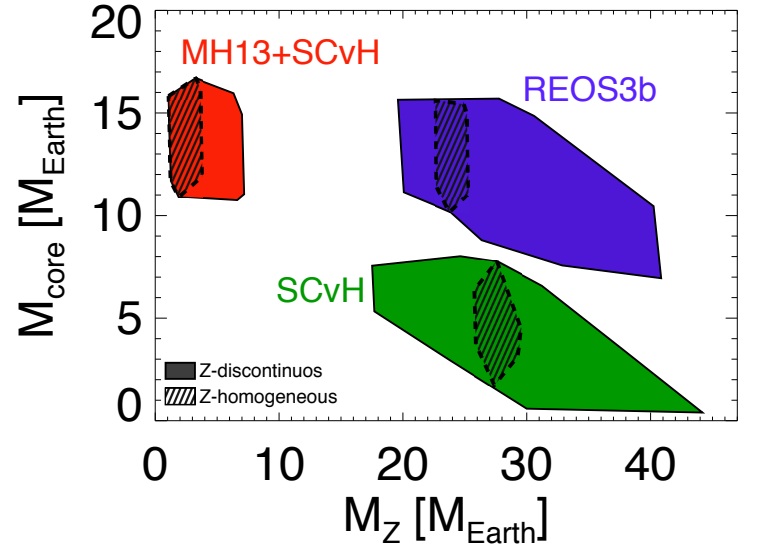

Fig. 9. Areas in the mass of the core and heavy elements space correspond to solutions found within $2 \sigma$ and different equations of state for $\mathrm{H}$ and $\mathrm{He}$ : $\mathrm{SCvH}$ (green), $\mathrm{MH} 13+\mathrm{SCvH}$ (red) and REOS3b (blue area). Results found with $Z$-homogeneous are the areas within the dashed lines and correspond to a subgroup of the $Z$-discontinuous solutions (we maintain this in all figures from now on).

captions. We considered uncertainties in the averaged helium mass mixing-ratio, the atmospheric helium mass mixing-ratio, the mass mixing-ratio of rocks and ices, and the ice fraction in the core. Due to these uncertainties, our range of potential solutions covers an area in the $M_{\text {core }}-M_{Z}$ diagram. In addition, we explored different values of $J_{2}$ and $J_{4}$ (see Table 1), different equations of state for heavy elements, and we changed the location of the helium phase transition to explore the sensitivity of the results to different model input parameters.

We ran optimizations for the three different equations of state for hydrogen and helium explored in this work. It is important to note that we started each one of these runs with the same model, the same initial conditions, and the same space of parameters to vary, but changed only the equation of state for hydrogen and helium. Figure 9 shows that the internal structure of Jupiter is extremely sensitive to the adopted equation of state, as expected from the differences in thermal profiles shown in static models (Sect. 4.1). The different equations of state lead to a completely different set of solutions that do not intersect with each other. While $\mathrm{SCvH}$ leads to an interior of Jupiter with a small core and a large amount of heavy elements, results found with REOS $3 b$ indicate a much higher mass of heavy elements in general: a large core and a large abundance of heavy elements, and $\mathrm{MH} 13+\mathrm{SCvH}$ leads to a large core and a very small amount of heavy elements in the interior of Jupiter.

Figure 10 shows that the structure of Jupiter is also sensitive to the equation of state for heavy elements adopted in the model (Sect. 3.3).

For REOS3b, both $M_{\text {core }}$ and $M_{Z}$ become lower when we use the dry sand SESAME, while the mass of heavy elements increases when we use $\mathrm{H}_{2} \mathrm{O}$ SESAME, when compared with results found with $\mathrm{H}_{2} \mathrm{O}$ NIST EOS. For $\mathrm{SCvH} M_{\text {core }}$ is lower for dry sand SESAME and $M_{Z}$ is also lower for the same core masses in comparison to results found with $\mathrm{H}_{2} \mathrm{O}$ NIST EOS. $\mathrm{MH} 13+\mathrm{SCvH}$ is less sensitive to changes in the EOS for heavy elements.

We tested the sensitivity of the results to different $P_{\text {sep }}$. Figure 11 shows that when $P_{\text {sep }}$ moves from higher (4 Mbar) to lower pressures $(0.8 \mathrm{Mbar})$, more solids are found in the core. 


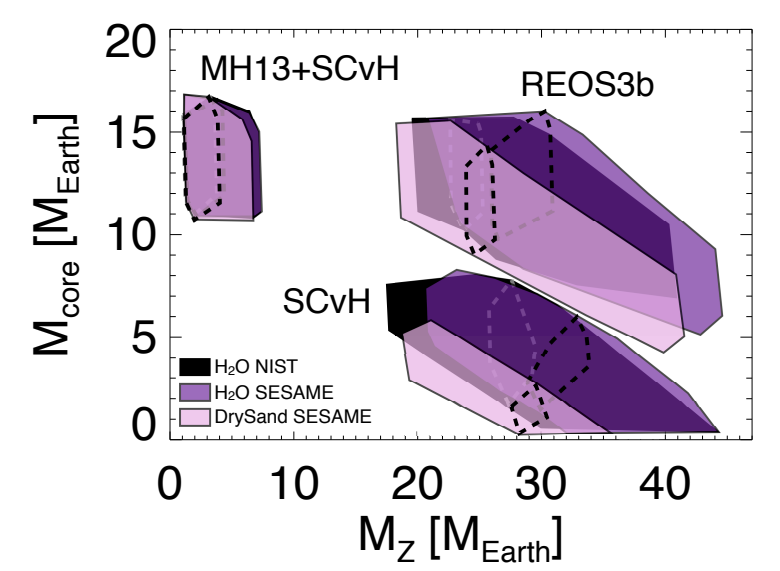

Fig. 10. Space of solutions obtained with different equations of state for $\mathrm{H}$ and $\mathrm{He}$ and for heavy elements. The equations of state for hydrogen and helium are indicated above the areas, and the equations of state of heavy elements have different colors: $\mathrm{H}_{2} \mathrm{O}$ NIST is black, Drysand SESAME is purple, and $\mathrm{H}_{2} \mathrm{O}$ NIST is pink.

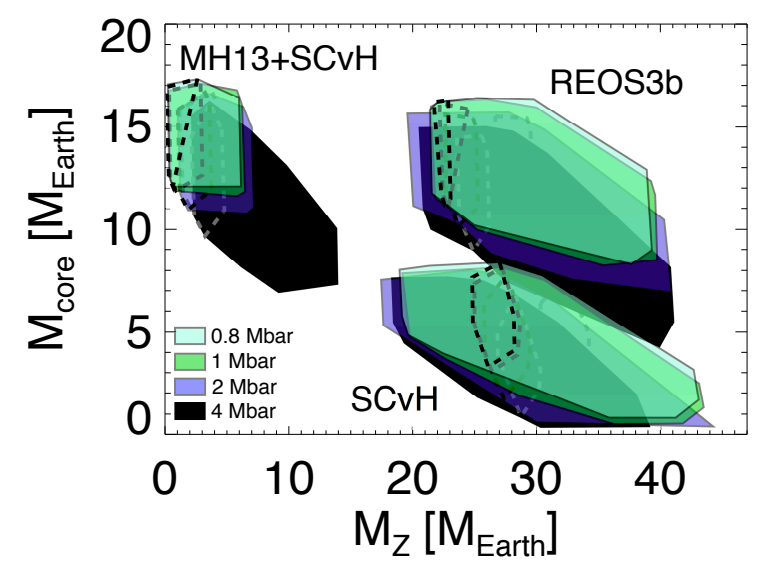

Fig. 11. Results of optimization models with different equations of state for $\mathrm{H}$ and $\mathrm{He}$ and changing the location of the $P_{\mathrm{Sep}}: 4 \mathrm{Mbar}$ (black), 2 Mbar (blue), 1 Mbar (green), and 0.8 Mbar (light blue).

\subsection{Discussion: sensitivity to internal energy calculations}

The REOS. 3 tables were constructed with a different scheme than $\mathrm{SCvH}$ tables. Their internal energies are not the same, not even in the $\mathrm{H}_{2}$ regime. We constructed REOS3b tables by changing the zero point of the specific internal energy to coincide with SCvH values at $T=60 \mathrm{~K}$ and $\rho=10^{-3} \mathrm{~g} / \mathrm{cm}^{3}$, but the difference between the tables differs at different temperatures. Results by Militzer \& Ceperley (2001), Militzer (2013), Militzer \& Hubbard (2013) also show differences with SCvH internal energies. The authors found that the $\mathrm{SCvH}$ model considers lower temperature intervals for the ionization of hydrogen atoms, which causes the discrepancy with their internal energies results.

To test the sensitivity of the internal structure calculations to differences in the internal energy derivation, we calculated a second equation of state based on the REOS.3 results, in which we calculated the difference between REOS.3 and SCvH and shifted the internal energies at all densities accordingly so that they coincided at $\rho=10^{-3} \mathrm{~g} / \mathrm{cm}^{3}$ for all temperatures. We then calculated the entropy for each point of the table and performed static and optimized calculations. We called these new tables REOS3sc (shown in Appendix A). Figure 12 shows the internal structure of Jupiter calculated with REOS3b and REOSsc. The differences

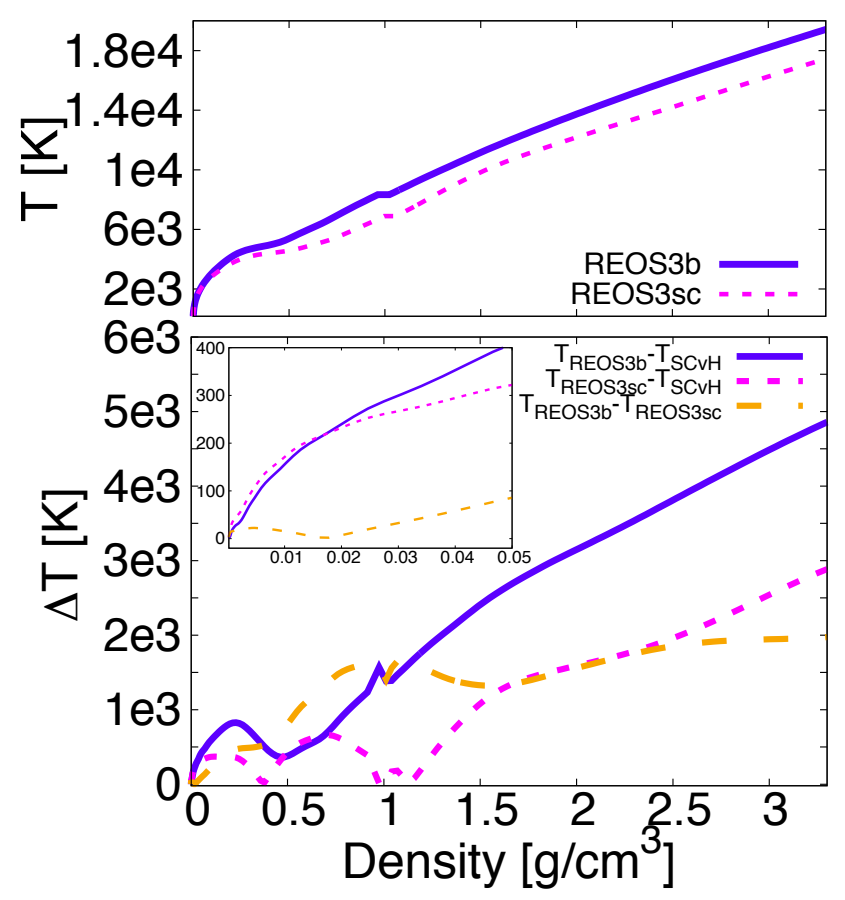

Fig. 12. Thermal profile calculated for Jupiter when using two equations of state derived using different internal energies. Top panel shows temperature vs. density, where blue is REOS $3 \mathrm{~b}$ and dashed magenta line was obtained with our test case the REOS3sc eos. Lower panel shows the differences in temperatures derived with the different equations of state: blue line is the difference between REOS3b and $\mathrm{SCvH}$, dashed magenta line is the difference between REOS3sc and SCvH and orange line is the difference between REOS3b and REOS3sc.

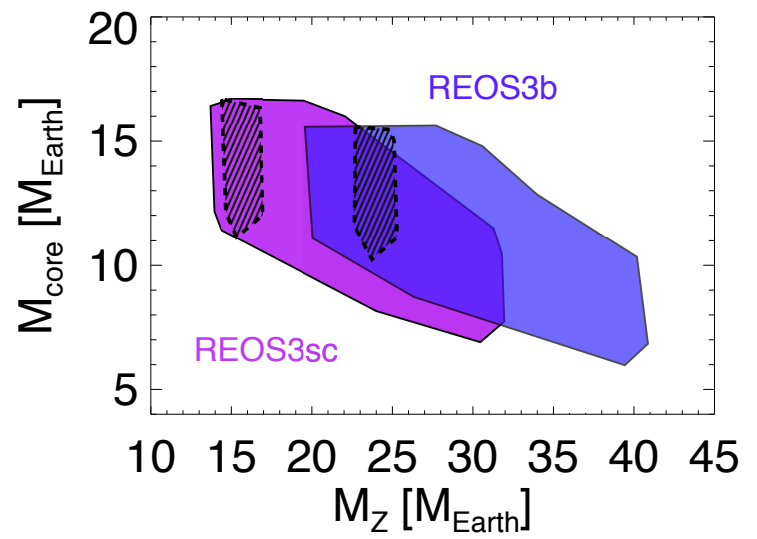

Fig. 13. Differences between the results obtained with REOS3b and REOS3sc equations of state.

in internal energy lead to a difference in the entropies that affects the thermal profile.

The different temperatures in the interior of the planet lead to a different core mass and mass of heavy elements derived in the optimized calculations. Figure 13 shows the solutions found with both equations of state, which shows that results are very sensitive to the internal energy and entropy calculations.

\section{Conclusions}

The reservoir of heavy elements in Jupiter is key to understanding the origin of our solar system. Nevertheless, the distribution and amount of heavy elements in the planet interior is difficult to constrain and degeneracies arise depending on the assumed 
observational constraints and model parameters in interior structure calculations. We presented optimized models of Jupiter, where the mass of the core and the mass of heavy elements are adjusted to reproduce the Jupiter radius, $J_{2}$ and $J_{4}$. We showed the drastic change in our solutions with the EOS for hydrogen and helium and also explored the sensitivity to heavy elements equations of state, to the separation between metallic and molecular envelope, and to the distribution of heavy elements in the interior of Jupiter.

We adopted two different models for Jupiter. The two scenarios considered helium phase separation and correspondingly different helium abundances in the outer and deeper layer. The difference is in the heavy elements distribution: one scenario has a homogeneous distribution of heavy elements, and its mass mixing-ratio is adjusted according to the observables. In the second scenario, Jupiter has different compositions of heavy elements in the two layers, and the difference in the abundance in the outer and deeper envelope $(\Delta Z)$ is adjusted to find solutions that best reproduce observational data of Jupiter. Allowing a change in heavy elements between the two layers adds a degree of freedom to the problem that grants more solutions in the $M_{Z}-M_{\text {core }}$ space. The pressure at which the separation between the two envelope layers occurs affects the solutions. This separation occurs between 0.8 and $4 \mathrm{Mbar}$, according to the helium rain studies of Morales et al. (2013). We found that $M_{Z}$ decreases and $M_{\text {core }}$ increases when $P_{\text {sep }}$ moves from high to low pressures.

Based on the works by Saumon et al. (1995), Militzer \& Hubbard 2013, Becker et al. (2014), we explore hydrogen and helium equations of state and showed that significant differences remain in these EOSs, although they match experimental data obtained by compression experiments along a Hugoniot. Some of the differences come from internal energy and entropy calculations. We showed that small changes in the internal energy lead to differences in the entropy that is calculated, which in turn affects the thermal profile and the estimation of the mass of the core and heavy elements. This explains differences seen in recently published interior models of the planet. The internal structure of Jupiter has a much higher temperature when REOS3b is used than what is derived with SCvH. For densities $\rho>0.22246 \mathrm{~g} / \mathrm{cm}^{3}, \mathrm{MH} 13+\mathrm{SCvH}$ leads to much lower temperatures than the other two EOS. These differences in the thermal structure lead to differences in the derived $M_{\text {core }}$ and $M_{Z}$. $\mathrm{MH} 13+\mathrm{SCvH}$ allows higher $M_{\text {core }}$ and lower $M_{Z}$, while REOS3b has higher $M_{\text {core }}$ but similar $M_{Z}$ as results found with $\mathrm{SCvH}$.

In our baseline simulations, $\mathrm{MH} 13+\mathrm{SCvH}$ leads to $M_{\text {core }}$ between 11 and $17 M_{\text {Earth }}$, in agreement with results by Militzer \& Hubbard 2013 and the preferred model of Hubbard \& Militzer (2016). REOS3b leads to $M_{\text {core }}$ between 7 and $16 M_{\text {Earth }}$, higher than estimates by Nettelmann et al. (2012) and Becker et al. (2014). While their preferred model has $P_{\text {sep }} \geq 4$ Mbar, our models place the separation between $Z_{\text {atm }}$ and $Z_{\text {deep }}$ in the same place as the helium phase transition, between 0.8 and $4 \mathrm{Mbar}$ (Morales et al. 2013), and the baseline simulations have $P_{\text {sep }}=$ 2 Mbar. When comparing the results at $P_{\text {sep }}=4 \mathrm{Mbar}$, we find a lower limit for the mass of the core of $4 M_{\text {Earth }}$, consistent with the small core hypothesis shown by Nettelmann et al. (2012) and Becker et al. (2014) for the same case. Other small differences are due to different model parameters such as the temperature at the 1 bar limit, the equation of state used for solids, and differences in the entropy calculation.

The equation of state for the heavy elements is also relevant. We studied three different equations of state for rocks and water. Dry sand SESAME (Lyon \& Johnson 1992) allows a lower $M_{\text {core }}$, while $M_{Z}$ increases when we used $\mathrm{H}_{2} \mathrm{O}$ SESAME
(Lyon \& Johnson 1992) when compared with solutions obtained with the hot water NIST EOS (Valencia et al. 2013).

Our results help in the interpretation of observational data of Jupiter. Its gravitational moments changed from the first preJuno data (Campbell \& Synnott 1985) to the constraints we have today (Jacobson 2013). They also change according to the dynamics and rotation of Jupiter that are adopted in the model. Given the relatively large scatter in the gravitational moments of Jupiter inferred between 1985 and today, in our baseline simulations we chose to use conservative $2 \sigma$ error bars based on the published value of Campbell \& Synnott (1985), which encompasses all of these values. We also showed that different $J_{\mathrm{S}}$ lead to different estimates of the core and heavy elements masses, with a difference of up to $4 M_{\text {Earth }}$ in $M_{\text {core }}$ and $\sim 6 M_{\text {Earth }}$ in $M_{Z}$ for REOS3b and $\mathrm{SCvH}$. Our preferred results have larger $J_{6}$ than previously published. The Juno mission will provide more accurate data that will improve our knowledge of the internal structure of Jupiter.

Acknowledgements. Y.M. greatly appreciates the CNES post-doctoral fellowship program. Y.M. and T.G. acknowledge the support of CNES for travel funding. Computations have been done on the "Mesocentre SIGAMM" machine, hosted by the Observatoire de la Côte d'Azur. We thank Bill Hubbard and Naor Movshovitz for valuable comments and for providing model comparisons to estimate the error in the $J_{\mathrm{s}}$ calculation. We also thank Andreas Becker, Nadine Nettelmann, and Burkhard Militzer for fruitful discussions regarding equations of state.

\section{References}

Atkinson, D. H., Pollack, J. B., \& Seiff, A. 1998, J. Geophys. Res., 103, 22911 Bahcall, J. N., \& Pinsonneault, M. H. 1995, Rev. Mod. Phys., 67, 781

Baraffe, I., Chabrier, G., Fortney, J., \& Sotin, C. 2014, Protostars and Planets VI, 763

Becker, A., Winfried, L., Fortney, J., et al. 2014, ApJS, 215, 21

Belov, S. I., Boriskov, G. V., Bykov, A. I., et al. 2002, Sov. J. Exp. Theor. Phys Lett., 76, 433

Brygoo, S., Millot, M., Loubeyre, P., et al. 2015, J. Appl. Phys., 118, 195901

Boriskov, G. V., Bykov, A. I., Il'Kaev, R. I., et al. 2003, Doklady Physics, 48, 553

Caillabet, L., Mazevet, S., \& Loubeyre, P. 2011, Phys. Rev. B, 83, 094101

Campbell, J. K., Synnott, S. P. 1985, AJ, 90, 364

Celliers, P. M., Loubeyre, P., Eggert, J. H., et al. 2010, Phys. Rev. Lett., 104, 184503

Collins, G. W., da Silva, L. B., Celliers, P., et al. 1998, Science, 281, 1178

Eggert, J., Brygoo, S., Loubeyre, P., et al. 2008, Phys. Rev. Lett., 100, 124503

Fortney, J. J., \& Nettelmann, N. 2010, Space Sci. Rev., 152, 423

French, M., Mattsson, T. R., Nettelmann, N., \& Redmer, R. 2009, Phys. Rev. B, 79, 054107

Galanti, E., \& Kaspi, Y. 2016, ApJ, 820, 91

Grishechkin, S. K., Gruzdev, S. K., Gryaznov, V. K., et al. 2004, Sov. J. Exp. Theor. Phys. Lett., 80, 398

Guillot, T. 1999, Planet. Space Sci., 47, 1183

Guillot, T., \& Gautier, D. 2015, in Giant Planets, Treatise on Geophysics, 2nd edn., ed. G. Schubert (Oxford: Elsevier), Vol. 10, 529

Guillot, T., \& Morel, P. 1995, A\&AS, 109, 109

Guillot, T., Chabrier, G., Morel, P., \& Gautier, D. 1994, Icarus, 112, 354

Guillot, T., Stevenson, D. J., Hubbard, W. B., \& Saumon, D. 2004, in Cambridge Planetary Science, Vol. 1 (CUP), 35

Hicks, D. G., Boehly, T. R., Celliers, P. M., et al. 2009, Phys. Rev. B, 79, 014112 Holmes, N. C., Ross, M., \& Nellis, W. J. 1995, Phys. Rev. B, 52, 15835

Hubbard, W. B. 1982, Icarus 52, 509

Hubbard, W. B. 2012, ApJ, 756, L15

Hubbard, W. B. 2013, ApJ, 768, 43

Hubbard, W. B., \& Militzer, B. 2016, ApJ, 820, 80

Kaspi, Y., Hubbard, W. B., Showman, A. P., \& Flierl, G. R. 2010 , Geophys. Res. Lett., 37, L01204

Knudson, M. D., Hanson, D. L., Bailey, J. E., et al. 2004, Phys. Rev. B, 69, 144209

Leconte, J., \& Chabrier, G. 2012, A\&A, 540, A20

Lindal, G. F., Mar. 1992, AJ, 103, 967

Loubeyre, P., Brygoo, S., Eggert, J., et al. 2012, Phys. Rev. B, 86, 144115 
Lyon, S., \& Johnson, J. , 1992, LANL Report LA-UR-92-3407, Los Alamos Mankovich, C., Fortney, J. J., \& Moore, K. L. 2016, ApJ, 832, 113

Militzer, B. 2006, Phys. Rev. Lett., 97, 175501

Militzer, B. 2009, Phys. Rev. B, 79, 155105

Militzer, B. 2013, Phys. Rev. B, 87, 014202

Militzer, B., \& Ceperley, D. M. 2001, Phys. Rev. E, 63, 066404

Militzer, B., \& Hubbard, W. B. 2009, Ap\&SS, 322, 129

Militzer, B., \& Hubbard, W. B. 2013, ApJ, 774, 148

Militzer, B., Hubbard, W. B., Vorberger, J., Tamblyn, I., \& Bonev, S. A. 2008 ApJ, 688, L45

Morales, M. A., Hamel, S., Caspersen, K., \& Schwegler, E. 2013, Phys. Rev. B 87,174105

Nellis, W. J., Mitchell, A. C., van Thiel, M., et al. 1983, J. Chem. Phys., 79, 1480
Nellis, W. J., Holmes, N. C., Mitchell, A. C., et al. 1984, Phys. Rev. Lett., 53, 1248

Nettelmann, N., Holst, B., Kietzmann, A., et al. 2008, ApJ, 683, 1217

Nettelmann, N., Becker, A., Holst, B., \& Redmer, R. 2012, ApJ, 750, 52

Nettelmann, N., Fortney, J. J., Moore, K., \& Mankovich, C. 2015, MNRAS, 447, 3422

Saul, A., \& Wagner, W. 1989, J. Phys. Chem. Ref. Data, 18, 1537

Saumon, D., \& Guillot, T. 2004, ApJ, 609, 1170

Saumon, D., Chabrier, G., \& van Horn, H. M. 1995, ApJS, 99, 713

Valencia, D., Guillot, T., Parmentier, V., \& Freedman, R. S. 2013, ApJ, 775, 10

Vazan, A., Helled, R., Podolak, M., \& Kovetz, A. 2016, ApJ, 829, 118

von Zahn, U., Hunten, D. M., \& Lehmacher, G. 1998, J. Geophys. Res., 103, 22815 


\section{Appendix A: Equations of state}

We present the equations of state derived in this paper. We note that the equations of state were tested and used only in a restricted range of pressures $\left(10^{6}\right.$ to $\left.10^{14} \mathrm{dyn} / \mathrm{cm}^{2}\right)$ and temperatures $\left(100\right.$ to $\left.10^{5} \mathrm{~K}\right)$ relevant for modeling the internal structure of Jupiter. There are some deviations between the entropies we calculated and those in SCvH table for $\log (s)<8.6$ in the hydrogen tables and for $\log (s)<8.2$ and densities $\log (\rho)<-5$ in the helium tables.

All the tables in this Appendix are available in their entirety at the CDS. A portion is shown here for guidance regarding their form and content.

Table A.1. MH13+SCvH table for hydrogen.

\begin{tabular}{cccc}
\hline \hline $\log (P)\left[\mathrm{dyn} / \mathrm{cm}^{2}\right]$ & $\log (T)[\mathrm{K}]$ & $\log (\rho)\left[\mathrm{g} / \mathrm{cm}^{3}\right]$ & $\log (s)[\mathrm{erg} / \mathrm{gK}]$ \\
\hline $0.400000000000 \mathrm{E}+01$ & $0.225000000000 \mathrm{E}+01$ & $-0.586540289041 \mathrm{E}+01$ & $0.892592538942 \mathrm{E}+01$ \\
$0.415000000000 \mathrm{E}+01$ & $0.225000000000 \mathrm{E}+01$ & $-0.571540399975 \mathrm{E}+01$ & $0.891852390428 \mathrm{E}+01$ \\
$0.430000000000 \mathrm{E}+01$ & $0.225000000000 \mathrm{E}+01$ & $-0.556540554822 \mathrm{E}+01$ & $0.891099450182 \mathrm{E}+01$ \\
$0.445000000000 \mathrm{E}+01$ & $0.225000000000 \mathrm{E}+01$ & $-0.541540769999 \mathrm{E}+01$ & $0.890333324035 \mathrm{E}+01$ \\
$0.460000000000 \mathrm{E}+01$ & $0.225000000000 \mathrm{E}+01$ & $-0.526541076753 \mathrm{E}+01$ & $0.889553366864 \mathrm{E}+01$ \\
$0.475000000000 \mathrm{E}+01$ & $0.225000000000 \mathrm{E}+01$ & $-0.511541508881 \mathrm{E}+01$ & $0.888759147622 \mathrm{E}+01$ \\
$0.490000000000 \mathrm{E}+01$ & $0.225000000000 \mathrm{E}+01$ & $-0.496542119569 \mathrm{E}+01$ & $0.887950126501 \mathrm{E}+01$ \\
$0.505000000000 \mathrm{E}+01$ & $0.225000000000 \mathrm{E}+01$ & $-0.481542982137 \mathrm{E}+01$ & $0.887125727110 \mathrm{E}+01$ \\
\hline
\end{tabular}

Table A.2. REOS $3 b$ table for hydrogen.

\begin{tabular}{cccc}
\hline \hline $\log (P)\left[\mathrm{dyn} / \mathrm{cm}^{2}\right]$ & $\log (T)[\mathrm{K}]$ & $\log (\rho)\left[\mathrm{g} / \mathrm{cm}^{3}\right]$ & $\log (s)[\mathrm{erg} / \mathrm{gK}]$ \\
\hline $0.750000000000 \mathrm{E}+01$ & $0.177815127000 \mathrm{E}+01$ & $-0.174398792000 \mathrm{E}+01$ & $0.851118183000 \mathrm{E}+01$ \\
$0.757499981000 \mathrm{E}+01$ & $0.177815127000 \mathrm{E}+01$ & $-0.160381353000 \mathrm{E}+01$ & $0.848558712000 \mathrm{E}+01$ \\
$0.764999962000 \mathrm{E}+01$ & $0.177815127000 \mathrm{E}+01$ & $-0.140540540000 \mathrm{E}+01$ & $0.843856716000 \mathrm{E}+01$ \\
$0.772499943000 \mathrm{E}+01$ & $0.177815127000 \mathrm{E}+01$ & $-0.130222011000 \mathrm{E}+01$ & $0.840594292000 \mathrm{E}+01$ \\
$0.779999924000 \mathrm{E}+01$ & $0.177815127000 \mathrm{E}+01$ & $-0.125477469000 \mathrm{E}+01$ & $0.838819885000 \mathrm{E}+01$ \\
$0.787499905000 \mathrm{E}+01$ & $0.177815127000 \mathrm{E}+01$ & $-0.122213173000 \mathrm{E}+01$ & $0.837435246000 \mathrm{E}+01$ \\
$0.794999886000 \mathrm{E}+01$ & $0.177815127000 \mathrm{E}+01$ & $-0.119594634000 \mathrm{E}+01$ & $0.836197186000 \mathrm{E}+01$ \\
$0.802499866000 \mathrm{E}+01$ & $0.177815127000 \mathrm{E}+01$ & $-0.117360163000 \mathrm{E}+01$ & $0.835033607000 \mathrm{E}+01$ \\
\hline
\end{tabular}

Table A.3. REOS3b table for helium.

\begin{tabular}{cccc}
\hline \hline $\log (P)\left[\mathrm{dyn} / \mathrm{cm}^{2}\right]$ & $\log (T)[\mathrm{K}]$ & $\log (\rho)\left[\mathrm{g} / \mathrm{cm}^{3}\right]$ & $\log (s)[\mathrm{erg} / \mathrm{gK}]$ \\
\hline $0.450000000000 \mathrm{E}+01$ & $0.177815127000 \mathrm{E}+01$ & $-0.459566355000 \mathrm{E}+01$ & $0.848160362000 \mathrm{E}+01$ \\
$0.457499981000 \mathrm{E}+01$ & $0.177815127000 \mathrm{E}+01$ & $-0.452066803000 \mathrm{E}+01$ & $0.847642231000 \mathrm{E}+01$ \\
$0.464999962000 \mathrm{E}+01$ & $0.177815127000 \mathrm{E}+01$ & $-0.444567299000 \mathrm{E}+01$ & $0.847117710000 \mathrm{E}+01$ \\
$0.472499943000 \mathrm{E}+01$ & $0.177815127000 \mathrm{E}+01$ & $-0.437067795000 \mathrm{E}+01$ & $0.846586895000 \mathrm{E}+01$ \\
$0.479999924000 \mathrm{E}+01$ & $0.177815127000 \mathrm{E}+01$ & $-0.429568529000 \mathrm{E}+01$ & $0.846049404000 \mathrm{E}+01$ \\
$0.487499905000 \mathrm{E}+01$ & $0.177815127000 \mathrm{E}+01$ & $-0.422069359000 \mathrm{E}+01$ & $0.845505142000 \mathrm{E}+01$ \\
$0.494999886000 \mathrm{E}+01$ & $0.177815127000 \mathrm{E}+01$ & $-0.414570236000 \mathrm{E}+01$ & $0.844953823000 \mathrm{E}+01$ \\
$0.502499866000 \mathrm{E}+01$ & $0.177815127000 \mathrm{E}+01$ & $-0.407071352000 \mathrm{E}+01$ & $0.844405270000 \mathrm{E}+01$ \\
\hline
\end{tabular}


Table A.4. REOS3sc table for hydrogen.

\begin{tabular}{cccc}
\hline \hline $\log (P)\left[\mathrm{dyn} / \mathrm{cm}^{2}\right]$ & $\log (T)[\mathrm{K}]$ & $\log (\rho)\left[\mathrm{g} / \mathrm{cm}^{3}\right]$ & $\log (s)[\mathrm{erg} / \mathrm{gK}]$ \\
\hline $0.750000000000 \mathrm{E}+01$ & $0.177815127000 \mathrm{E}+01$ & $-0.174398792000 \mathrm{E}+01$ & $0.849107647000 \mathrm{E}+01$ \\
$0.757499981000 \mathrm{E}+01$ & $0.177815127000 \mathrm{E}+01$ & $-0.160381353000 \mathrm{E}+01$ & $0.846421719000 \mathrm{E}+01$ \\
$0.764999962000 \mathrm{E}+01$ & $0.177815127000 \mathrm{E}+01$ & $-0.140540540000 \mathrm{E}+01$ & $0.841470623000 \mathrm{E}+01$ \\
$0.772499943000 \mathrm{E}+01$ & $0.177815127000 \mathrm{E}+01$ & $-0.130222011000 \mathrm{E}+01$ & $0.838014793000 \mathrm{E}+01$ \\
$0.779999924000 \mathrm{E}+01$ & $0.177815127000 \mathrm{E}+01$ & $-0.125477469000 \mathrm{E}+01$ & $0.836175156000 \mathrm{E}+01$ \\
$0.787499905000 \mathrm{E}+01$ & $0.177815127000 \mathrm{E}+01$ & $-0.122213173000 \mathrm{E}+01$ & $0.834656525000 \mathrm{E}+01$ \\
$0.794999886000 \mathrm{E}+01$ & $0.177815127000 \mathrm{E}+01$ & $-0.119594634000 \mathrm{E}+01$ & $0.833196449000 \mathrm{E}+01$ \\
$0.802499866000 \mathrm{E}+01$ & $0.177815127000 \mathrm{E}+01$ & $-0.117360163000 \mathrm{E}+01$ & $0.831901360000 \mathrm{E}+01$ \\
\hline
\end{tabular}

Table A.5. REOS3sc table for helium.

\begin{tabular}{cccc}
\hline \hline $\log (P)\left[\mathrm{dyn} / \mathrm{cm}^{2}\right]$ & $\log (T)[\mathrm{K}]$ & $\log (\rho)\left[\mathrm{g} / \mathrm{cm}^{3}\right]$ & $\log (s)[\mathrm{erg} / \mathrm{gK}]$ \\
\hline $0.450000000000 \mathrm{E}+01$ & $0.177815127000 \mathrm{E}+01$ & $-0.459566355000 \mathrm{E}+01$ & $0.849313259000 \mathrm{E}+01$ \\
$0.457499981000 \mathrm{E}+01$ & $0.177815127000 \mathrm{E}+01$ & $-0.452066803000 \mathrm{E}+01$ & $0.848808765000 \mathrm{E}+01$ \\
$0.464999962000 \mathrm{E}+01$ & $0.177815127000 \mathrm{E}+01$ & $-0.444567299000 \mathrm{E}+01$ & $0.848298264000 \mathrm{E}+01$ \\
$0.472499943000 \mathrm{E}+01$ & $0.177815127000 \mathrm{E}+01$ & $-0.437067795000 \mathrm{E}+01$ & $0.847781658000 \mathrm{E}+01$ \\
$0.479999924000 \mathrm{E}+01$ & $0.177815127000 \mathrm{E}+01$ & $-0.429568529000 \mathrm{E}+01$ & $0.847258949000 \mathrm{E}+01$ \\
$0.487499905000 \mathrm{E}+01$ & $0.177815127000 \mathrm{E}+01$ & $-0.422069359000 \mathrm{E}+01$ & $0.846729851000 \mathrm{E}+01$ \\
$0.494999886000 \mathrm{E}+01$ & $0.177815127000 \mathrm{E}+01$ & $-0.414570236000 \mathrm{E}+01$ & $0.846194172000 \mathrm{E}+01$ \\
$0.502499866000 \mathrm{E}+01$ & $0.177815127000 \mathrm{E}+01$ & $-0.407071352000 \mathrm{E}+01$ & $0.845651817000 \mathrm{E}+01$ \\
\hline
\end{tabular}

